\title{
USO DE SOFTWARE LIBRE Y DE INTERNET COMO HERRAMIENTAS DE APOYO PARA EL APRENDIZAJE
}

\author{
(USE OF FREE SOFTWARE AND INTERNET LIKE TOOLS OF SUPPORT FOR LEARNING)
}

Carlos Lizárraga Celaya

Sara Lorelí Díaz Martínez

Universidad de Sonora (México)

\section{RESUMEN}

El presente trabajo describe el uso de herramientas de tecnologías de la información y la comunicación como apoyo al proceso de aprendizaje, las cuales están basadas en aplicaciones propias de las computadoras con las características de Software Libre así como aplicaciones propias de la Web 2.o. Asimismo, se presenta la concepción de utilizar las computadoras como herramientas cognitivas para promover y desarrollar distintas habilidades del pensamiento. Posteriormente se enlistan por categorías algunos ejemplos de herramientas de la mente los cuales pueden ser descargados de internet o ser utilizados en línea.

Palabras clave: software libre, web 2.o, herramientas de la mente, herramientas cognitivas, ntic, tipos de pensamiento.

\begin{abstract}
This paper describes the use of information and communication technology tools to support learning. The tools presented are classified in the category of free software and web 2.0 applications. The view of using computers as cognitive tools to promote and develop skills of the different types of thinking. Later, some examples of mindtools are presented by categories that can be downloaded from internet or used on line directly.
\end{abstract}

Key words: free software, web 2.o, mindtools, cognitive tools, ict, types of thinking. 
Los estudiantes necesitan aprender a utilizar herramientas de las tecnologías de la información y la comunicación que les permitan dominar las habilidades de aprendizaje esenciales para la vida diaria y la productividad en el trabajo. Esta competencia se conoce como fluidez computacional y se puede definir como el interés, la actitud y la habilidad de las personas para utilizar eficazmente las tecnologías de la información y la comunicación (TIC's) con el objeto de acceder, manejar, integrar y evaluar información; construir nuevo conocimiento y comunicarse con otros con el propósito de ser participantes efectivos en la sociedad. Esta definición va más allá de una simple competencia técnica estrecha y limitada; debe ir acompañada de habilidades intelectuales de orden superior como pensamiento crítico y utilización inteligente, creativa y ética de las TIC's. Por otro lado, el rápido avance del desarrollo tecnológico conlleva también el constante desarrollo de sistemas que buscan dar respuesta a las diversas necesidades de los usuarios. Por lo anterior, pensar en comprar software propietario representa una importante inversión financiera que por otras necesidades no siempre se contemplan en los presupuestos ordinarios de las instituciones educativas con lo cual se podría alimentar el problema de la piratería a mayores dimensiones. Se busca entonces poner en contacto al estudiante ante la diversidad de herramientas de software que le ayudarán a ampliar su conocimiento sobre la existencia del mismo así como desarrollar las habilidades para absorber tecnológicamente nuevas herramientas, sin incurrir en faltas administrativas ni fomentar actitudes no éticas de utilizar programas sin autorización correspondiente.

Por su parte, las notorias ventajas en general del software libre (SL) respecto al software privativo en: dominio de mercado, confiabilidad, rendimiento, escalabilidad, seguridad y costo total de posesión ha promovido la aceptación del primero en muchos países, como alternativa más económica y potente. Asimismo se ha incrementado significativamente el volumen de proyectos de SL y código fuente abierto registrados en repositorios como SourceForge.net (más de ciento treinta mil, Octubre 2006), proyectos que cubren la mayoría de aplicaciones de software que se utilizan en el quehacer cotidiano.

\section{DEFINICIÓN DE SOFTWARE LIBRE}

El Movimiento de Software Libre surge a principios de 1980 con Richard Stallman del Laboratorio de Inteligencia Artificial del MIT. Crean en 1985 la Fundación GNU (http://www.gnu.org), para avanzar el movimiento y fomentar el desarrollo de software libre. Las computadoras sin la existencia de herramientas de software no son de utilidad, por ello se enfocarían los esfuerzos a desarrollar programas 
para hacer al hardware útil. Saliendo de la comunidad académica la concepción de software libre, mantiene el espíritu de que todo conocimiento debe ser compartido con el resto del mundo. Tanto el conocimiento, como el software, no deben tener propietarios (Stallman, 1994).

El software libre (Libre como en Libertad), cumple las cuatro libertades:

- Libertado, ejecutar el programa con cualquier propósito (privado, educativo, público, comercial, etc.).

- Libertad 1, estudiar y modificar el programa (para lo cuál es necesario poder acceder al código fuente).

- Libertad 2, copiar el programa de manera que se pueda ayudar al vecino o a cualquiera.

- Libertad 3, mejorar el programa, y hacer públicas las mejoras, de forma que se beneficie toda la comunidad.

Existe otra familia de software, el Software de Código Fuente Abierto (OpenSource.org), que en cierta forma coincide con algunos principios del Software Libre. Para nuestros propósitos, no distinguiremos las diferencias y lo incluiremos en una sola gran familia.

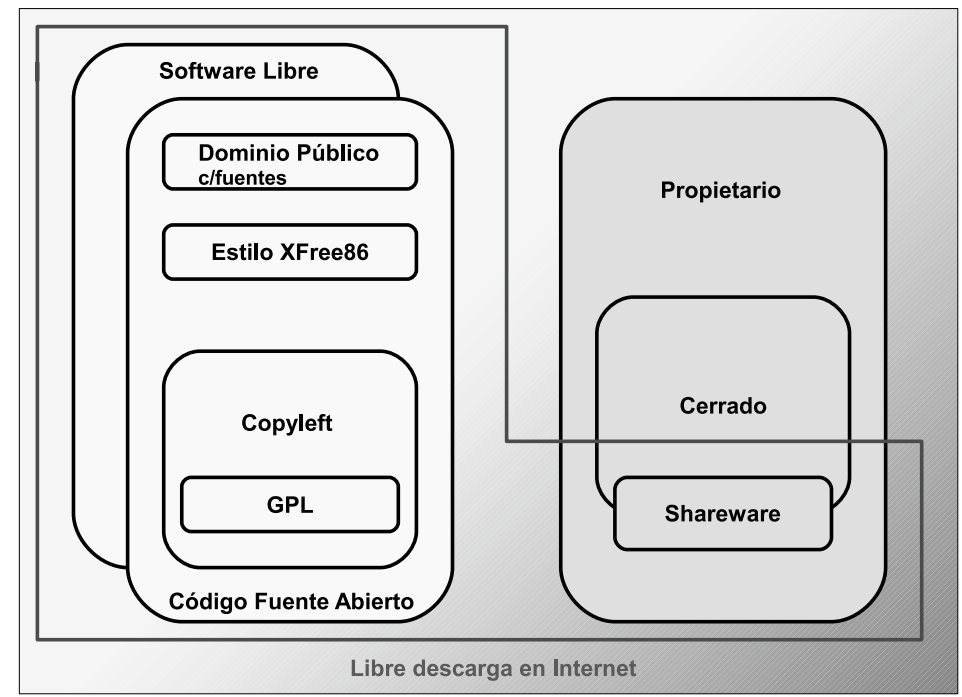

Figura 1. Diferencias entre software libre y propietario. 


\section{DIFERENCIAS ENTRE EL SOFTWARE LIBRE Y EL SOFTWARE PROPIETARIO}

Existe una diferencia marcada entre las licencias que protegen al Software Libre (Copyleft) y al Software Propietario o Privativo (Copyright). La Licencia Pública de la Fundación GNU (GPL) defiende al software que se originó libre para que mantenga su libertad y pueda seguir cumpliendo las cuatro libertades. La comunidad participa abiertamente en su desarrollo y lo modifica, lo adapta, lo enriquece y lo distribuye. Por el contrario, el software propietario o privativo, es cerrado, sólo unos cuantos tienen acceso al código fuente y sólo el propietario decide la dirección de desarrollo y la publicación de mejoras. Las licencias de software privativo nunca ceden el derecho de propiedad. Los usuarios sólo pagan por utilizarlo. Existen muchas variantes de licencias de software libre y de código fuente abierto, la licencia Copyleft más conocida es la licencia pública GNU (GPL) y se siguen desarrollando nuevas formas.

\section{VENTAJAS DEL USO DE SOFTWARE LIBRE}

Presentamos algunas ventajas de apoyar nuestras actividades cotidianas con herramientas de software libre:

- Está basado en el principio de colaboración comunitaria (principios de colaboración académica).

- No hay costo por licencias ni actualizaciones.

- No promueve acciones de piratería debido a que las licencias promueven la libre redistribución del software.

- Se encuentra soporte en una amplia comunidad en Internet.

- Existen para una variedad de plataformas (Windows, Mac, Linux, etc.).

- Permiten la portabilidad espacial y temporal del usuario. Las herramientas son independientes de las plataformas, no desaparecen, se mejoran con el tiempo.

- Existe una gran cantidad de virus para los productos de Microsoft. Estos virus no afectan a programas de Software Libre (aparecen del orden de 200 virus nuevos diarios).

- Sus actualizaciones son constantes y accesibles vía Internet.

- Son adaptables y configurables a las necesidades del usuario.

- Existe disponibilidad de gran diversidad de recursos ya que en SourceForge (http://sourceforge.net) se hospedan más de 130 mil proyectos de software libre (Octubre 2006). 


\section{EXPERIENCIAS EN EL USO DE SOFTWARE LIBRE Y NTIC}

A este respecto podemos tomar ventaja del desarrollo, cada vez mas vertiginoso en los últimos 25 años, de herramientas de software libre entre las que se encuentran:

- El desarrollo de plataformas tecnológicas para la colocación de materiales de apoyo a las actividades en línea de comunidades virtuales conformada por cientos o miles de usuarios.

- Herramientas de la mente tales como sistemas de ofimática, creación de mapas mentales, creación de diagramas de flujo, manipulación de imágenes, entre otros.

- Herramientas de comunicación diversas como correo electrónico, mensajería instantánea, mensajes privados, salas de conversaciones, foros de discusiones, por mencionar algunos.

- Acceso eficiente a recursos de información en línea (buscadores, metabuscadores, directorios temáticos, traductores, diccionarios, enciclopedias, uso de mapas, noticias, etc).

- Herramientas multimedia en línea para la publicación de trabajo individual o colaborativo (Weblogs, sistemas Wiki, páginas Web, podcasts).

- La posibilidad de enfrentar la problemática social asociada al Internet con respecto a seguridad informática (virus, gusanos o troyanos, software espía, correos no deseados, censura de la información, ciberderechos, comercio electrónico, entre muchos).

- Disponibilidad para localizar nuevos recursos de aplicaciones de software, de acuerdo a las necesidades de los usuarios.

\section{COMPUTADORA COMO HERRAMIENTA DE LA MENTE}

Las posibilidades del uso de la computadora se amplían a merced de los recursos y servicios que se encuentran en el Internet, tales como correo electrónico, chat, foros de discusión, intercambio, búsqueda y consulta, videoconferencias, trabajo colaborativo, entre otros y, los cuales a su vez de manera individual, plantean un esquema de interacción distinto (Pennock, 2000).

La aplicación de estas herramientas en el proceso educativo, están jerarquizadas por Escobar (Pennock, 2000) de la siguiente manera:

- Permiten la creación de un espacio personal de trabajo especialmente favorable donde la retroalimentación recibida permite monitorear el propio desarrollo. 
- Permiten exponer la información en diferentes formas de presentación, que incluyan gráficas, colores, animación y sonido.

- Permiten trabajar con tecnologías paralelas, como el $C D R O M$, videodiscos, sistemas de audio, digitalización de imágenes, videos pregrabados, etcétera.

- Posibilitan mayor interacción que muchos de los materiales tradicionales utilizados en la educación, aumentando las posibilidades de aprendizaje.

- La retroalimentación que se recibe por parte de los programas computacionales carece de juicios y cargas afectivas, es personal, no implica exhibiciones de error frente a otros y es directa hacia el error cometido.

Como se puede apreciar la computadora funge como una herramienta capaz de ofrecer distintos servicios conjugados en un solo elemento, lo que puede potenciar la interacción del individuo con este medio y el desarrollar habilidades que le permiten, mediante la combinación de diversas tecnologías, concebir nuevas formas tanto para acceder a la información como para desarrollar y proyectar el conocimiento (Díaz, 2004).

Las teorías actuales del aprendizaje recomiendan que los estudiantes, quienes de forma guiada construyen su aprendizaje deben trabajar en ambientes de aprendizaje que repliquen estructuras similares a las de las actividades en la vida real. Esto sugiere un cambio de paradigma que a veces no es posible llevar a cabo por completo. Una solución viable, es la de considerar diversas formas de la representación del conocimiento en las valoraciones. Para ello se le pide a los estudiantes, que representen de diversas formas lo que están aprendiendo, utilizando estrategias de aprendizaje activo, lo cual se puede facilitar con las tecnologías computacionales, utilizando a la computadora como una herramienta de la mente para representar su conocimiento (Jonassen, 1998, 2000, 2002).

Las herramientas de la mente promueven en el estudiante diversas formas de razonar sobre el contenido, es decir los hace pensar de diversas formas sobre lo que conocen y son aquellas herramientas con las que los sujetos aprenden y no de las que aprenden.

Las herramientas de cómputo, a diferencia de otras herramientas, pueden funcionar como socios intelectuales que comparten la responsabilidad cognitiva de llevar a cabo las tareas de apoyo al desarrollo del aprendizaje. Al utilizarlas, los aprendices se involucran en una variedad de pensamientos críticos, creativos y complejos, como lo es evaluar, sintetizar, imaginar, diseñar, resolver problemas, 
y tomar decisiones para las cuales muchas aplicaciones de cómputo han sido desarrolladas explícitamente para comprometer a los aprendices en pensamientos críticos y de las cuales hablaremos en lo subsecuente.

\section{LAS COMPUTADORAS COMO HERRAMIENTAS COGNITIVAS}

$\mathrm{Al}$ incorporar las computadoras como estas herramientas, utilizamos tecnologías de construcción que apoyan, guían y extienden el proceso de pensamiento de los usuarios. Estos elementos proveen formalismos estructurales, lógicos, causales, sistémicos, o visuales-espaciales en los que se apoyan distintos tipos de pensamientos y representación del conocimiento, de tal forma que le permite pensar al aprendiz en formas que no haría o podría.

Las herramientas de la mente son aplicaciones de software como bases de datos, hojas de cálculo, programas de redes semánticas, sistemas expertos, herramientas de modelación de sistemas, micromundos, herramientas hipermedia de autoría, video conferencias, que permiten al estudiante representar lo que han aprendido y lo que saben utilizando diversos formalismos. Al utilizar las herramientas de la mente, los aprendices se comprometen en una variedad de pensamientos críticos, creativos y complejos.

\section{HABILIDADES DEL PENSAMIENTO CRÍTICO}

El pensamiento crítico es un proceso intelectual disciplinado activo para conceptualizar, aplicar, analizar, sintetizar y/o evaluar información obtenida de la observación, experiencia, reflexión, razonamiento, o comunicación como guía de comportamiento (Scriven, 2004). El pensamiento crítico puede ser visualizado y formado de dos componentes:

- Un conjunto de información y habilidades para generar creencias y de procesamiento de la información.

- El hábito, basado en un compromiso intelectual, de usar esas habilidades para guiar el comportamiento.

El pensamiento crítico no es algo universal en un individuo. Cada persona se encuentra expuesta a experimentar episodios de pensamientos indisciplinados e irracionales. El desarrollo de habilidades para el pensamiento crítico y la disposición 
a ello, es una tarea que debe ejercitarse a lo largo de la vida. Mencionamos algunas actividades que apoyan al desarrollo de un pensamiento crítico sobre un tema:

- Evaluando:

- Valorando confiabilidad y utilidad

- Determinando criterios para juicio

- Priorizando

- Reconociendo falacias o errores

- Probando hipótesis

- Analizando:

- Reconociendo patrones

- Categorizando

- Identificando suposiciones

- Identificando las ideas principales

- Haciendo secuencias

\section{HABILIDADES DE PENSAMIENTO CREATIVO}

En los ambientes de educación formal, frecuentemente se hace mucho énfasis en las habilidades de análisis, promoviendo en el estudiante a la comprensión de conceptos, a seguir o crear un argumento lógico, a encontrar la solución de algo, a discernir entre trayectorias equivocadas y enfocarse en una correcta (Harris, 1998). Sin embargo, existe otro tipo de pensamiento, uno que se enfoca en explorar ideas, generar posibilidades, buscando diversas soluciones en vez de una sola. Ambos tipos de pensamientos son vitales para una vida profesional exitosa, pero sucede con frecuencia que esta última es a veces ignorada.

En la solución de problemas, ambos tipos de pensamientos son importantes. Primero debemos analizar el problema, luego generar soluciones posibles, después debemos escoger e implementar la mejor solución. Finalmente deberemos evaluar la efectividad de la solución. Esto ejemplifica, que el proceso revela una alternancia entre los dos tipos de pensamientos, el crítico y el creativo. En la práctica, ambos tipos de pensamientos operan juntos y realmente no son independientes el uno del otro. Mencionamos algunas actividades que fomentan el pensamiento creativo:

- Sintetizando:

- Pensando analógicamente

- Resumiendo las ideas principales en sus propias palabras 
- Haciendo hipótesis

- Realizando una planeación

- Imaginando:

- Expresando las ideas fluidamente

- Prediciendo resultados de condiciones

- Especulando

- Haciéndose preguntas

- Utilizando la intuición

- Elaborando:

- Expandiendo al añadir detalles o ejemplos

- Cambiando ideas para diferentes propósitos

- Aplicando a un contexto diferente

- Concretizando las ideas generales

\section{HABILIDADES DE PENSAMIENTO COMPLEJO}

Una de las metas del aprendizaje es que los aprendices puedan hacer algo con lo que han aprendido. Requieren extender y refinar su conocimiento aprendido. Hacer nuevas distinciones y más conexiones. El aprendizaje es efectivo cuando el conocimiento adquirido se utiliza para realizar tareas significativas.

Una persona realiza pensamientos complejos cuando ha desarrollado las habilidades de pensamiento necesarias para resolver problemas nuevos. Las siguientes son una serie de actividades que promueven el desarrollo de pensamientos complejos:

- Resolviendo problemas:

- Identificando el problema

- Investigando el problema

- Formulando el problema

- Encontrando alternativas

- Seleccionando una solución

- Construyendo la aceptación

- Diseñando:

- Imaginándose una meta

- Formulando una meta

- Inventando 
- Valorando

- Revisando un producto

- Tomando decisiones:

- Identificando un aspecto

- Generando alternativas

- Valorando las consecuencias

- Seleccionando

- Evaluando

\section{HERRAMIENTAS DE LA MENTE}

El papel de un sistema de computadora no es el de un maestro o experto, sino una herramienta cognitiva de extensión de la mente más que un agente de enseñanza. Por otro lado existen aplicaciones y herramientas más avanzadas y potentes que están en la Internet, que también pueden ser utilizadas, sobre todo aplicaciones de la siguiente generación: la Web 2.0 (O’Reilly, 2005; Polo, 2005). Algunas de estas aplicaciones sobre Internet son gratuitas para el usuario, gracias a otros esquemas de comercialización y pueden ser incorporadas para potenciar el alcance de la mente.

La tendencia actual es a la desaparición de las aplicaciones locales de escritorio a favor de las aplicaciones sobre la Internet, otorgando movilidad al usuario para acceder a su información y otras herramientas desde cualquier punto de la red, como ya sucede con la telefonía, la música, la televisión, almacenamiento de datos, comunicación con otros miembros de sus redes sociales, etc.

Existen diversas clases de herramientas de la mente, sobre el computador y sobre la Internet (Jonassen, 1998):

\section{Herramientas de organización semántica}

Son las que permiten al aprendiz analizar y organizar lo que saben o lo que están aprendiendo, entre estas tenemos:

\section{Bases de datos}

Además de contener grandes volúmenes de información, esos sistemas permiten al estudiante decidir el tipo de relaciones que se establecerán entre los datos que, a su misma vez, deciden que se almacenarán. Las bases de datos consisten en 
sistemas de archivación computarizada diseñados para acelerar el almacenamiento y recuperación de la información. La información se estructura en archivos que constan de matrices de registros y campos. Los registros contiene datos de un evento en específico y los campos describen las características puntuales de ese evento. Las bases de datos utilizan lógica booleana para acceder a la información relevante.

- OpenOffice Base (http://tuxiradical.bitacoras.com/index.php?id=125)

- Directorios Temáticos de Internet. http://dmoz.org/

- BD en línea (http://www.google.com, http://www.imdb.com, y más)

\section{Redes semánticas (mapas conceptuales)}

Estas redes permiten al estudiante extraer los elementos principales de un tópico y establecer sus relaciones con los elementos secundarios, obteniendo así una imagen que le permite ver claramente de forma gráfica la temática y los elementos que la componen. Una red semántica está compuesta por nodos donde cada uno representa un dato, que se relacionan entre sí por medio de enlaces (representados por flechas en el diagrama). Los nodos representan objetos o conceptos del dominio de problemas y las flechas sus relaciones o asociaciones. Estos mapas son las representaciones espaciales de ideas y sus interrelaciones que están almacenadas en la memoria, es decir, el llamado conocimiento estructural. Estos mapas visuales le permiten al aprendiz identificar los conceptos importantes, interrelacionar gráficamente esos conceptos en redes multidimensionales, y etiquetar las relaciones entre esos conceptos.

- Conzilla (http://www.conzilla.org/)

- Belvedere (http://belvedere.sourceforge.net/)

- FreeMind (http://freemind.sourceforge.net/wiki/index.php/Main Page)

- Kartoo (http://www.kartoo.com)

\section{Herramientas de modelaje dinámico}

Mientras la organización semántica ayuda a los aprendices a representar las relaciones semánticas entre las ideas, el modelaje dinámico les permite describir las relaciones dinámicas entre las ideas.

\section{Hojas de Cálculo}

A diferencia de las bases de datos, éstas permiten visualizar de primera mano sus capacidades de organización, así como mayor capacidad para realizar operaciones 
aritméticas. Una hoja de cálculo es un programa que permite manipular números dispuestos en forma de tablas. Habitualmente es posible realizar cálculos complejos con fórmulas y funciones y dibujar distintos tipos de gráficas. Las tablas de las hojas de cálculo comprenden los conceptos de celda, fila y columna, la intersección de una fila y una columna es llamada celda, las filas están etiquetadas por números consecutivos, mientras que las columnas están etiquetadas con letras consecutivas del abecedario.

- OpenOffice.org Calc (http://es.wikipedia.org/wiki/Calc)

- ThinkFree Calc (http://www.thinkfree.com/)

\section{Sistemas Expertos}

Los sistemas expertos son aquellos programas que se realizan haciendo explícito el conocimiento en ellos, mismos que tienen información específica de un dominio concreto y que realizan una tarea relativa a este dominio. Trabajan con inteligencia artificial simbólica, es decir, a través de ideas, imágenes, conceptos, etc. aunque debido a su naturaleza algorítmica se usen internamente instrucciones computables y estructuras de datos. El éxito de estos sistemas radica fundamentalmente en el conocimiento sobre el dominio que trata y su capacidad de aprendizaje. Lo anterior proporciona al sistema experto mayor información sobre el problema a tratar y su entorno, de forma que pueda generar y adaptar soluciones de forma más precisa, al tener un conocimiento más profundo sobre el tema, de forma similar a un experto especializado. El aprendizaje, inductivo o deductivo según corresponda, proporcionará al sistema mayor autonomía a la hora de abordar problemas totalmente desconocidos; pudiendo generar nuevo conocimiento partiendo del extraído inicialmente del experto o expertos humanos.

- F2w Helpdesk. Sistema para construir bases expertas de conocimiento sobre problemas y soluciones basados en categorías (http://f2w.sourceforge. net/).

- AI Planet. Herramienta para la construcción de mundos virtuales apoyados en Inteligencia Artificial (http://aiplanet.sourceforge.net/).

- A.L.I.C.E. Robot de chat construido en base a procesamiento de Lenguaje Natural y técnicas de Inteligencia Artificial (http://www.alicebot.org/).

- RoboCup Soccer Simulator. Herramienta para sistemas multi-agentes e Inteligencia Artificial que permite que dos equipos de 11 jugadores robots autónomos simulados jueguen fútbol soccer entre ellos (http://sserver. sourceforge.net/). 


\section{Modelación de sistemas}

El aprendizaje complejo requiere que los aprendices resuelvan tanto problemas complejos y mal planteados, como problemas sencillos. El aprendizaje complejo requiere que los aprendices desarrollen representaciones mentales de los fenómenos que se estudian.

- Celestia. Simulador de los cuerpos celestes. Permite la exploración del Universo en 3D (http://celestia.sourceforge.net/).

\section{Micromundos}

Son ambientes de aprendizaje exploratorios o espacios para descubrimiento, donde los participantes pueden navegar, crear o manipular objetos, y probar los efectos de unos sobre otros. Los micromundos contienen simulaciones constreñidas de los fenómenos de la vida real que permiten ser controladas por el participante.

- Proyecto de colonización de Marte (http://mars-sim.sourceforge.net/).

- Proyecto de Administración de una Universidad Virtual (http://www. virtual-u.org/).

\section{Herramientas de interpretación de la información}

El volumen y complejidad de la información crece a proporciones sin igual. Los aprendices requieren de herramientas que les ayuden a acceder y procesar la información.

\section{Herramientas de visualización}

Percibimos más información visualmente que con ningún otro sentido. No podemos expresar ideas visualmente, excepto en imágenes mentales o en sueños, las cuales no pueden ser compartidas, a excepción de usar programas de dibujo o para pintar. Debido a que no es posible transferir nuestras imágenes mentales a la computadora, han aparecido una serie de herramientas de visualización para razonar visualmente las cuales nos auxilian para representar y comunicar esas imágenes mentales de forma aproximada. 
- OpenOffice.Org Draw. Editor de gráficos vectoriales (http://es.wikipedia. org/wiki/OpenOffice.org Draw).

- Blender. Programa para dibujar en 3D (http://blender3d.org/).

- Inkscape. Programa de gráficos vectoriales (http://www.inkscape.org/)

- Art of Illusion. Programa para modelación y dibujo en 3D (http://aoi. sourceforge.net/).

- NASA World Wind. Herramienta para visualizar la superficie de la Tierra en 3D apoyado en imágenes de los satélites de NASA (http://worldwind.arc. nasa.gov/).

- Google Earth (http://earth.google.com/), Google Moon (http://moon. google.com/), Google Mars (http://mars.google.com/). Herramientas de Google para explorar la superficie terrestre, lunar y marciana, a partir de imágenes de NASA.

\section{Herramientas para la construcción de conocimiento}

Se utiliza el término construccionismo para describir el proceso de generación del conocimiento resultante de construir cosas. Cuando los aprendices trabajan de diseñadores de objetos aprenden más de los objetos que si solamente los hubieran estudiado.

\section{Hipermedia}

Consiste de nodos de información, que son las unidades básicas del almacenamiento de información. Puede consistir de una página de texto, una gráfica, un archivo de sonido, un clip de video o un documento completo. El usuario puede agregar o modificar elementos existentes para que un hipertexto represente una base de conocimiento dinámico que continua creciendo, representando nuevos y diferentes puntos de vista. Los sistemas de hipermedia han sido utilizados como sistemas de recuperación de información que los usuarios navegan, los aprendices pueden crear sus propias bases de conocimiento hipermedia que reflejen su propia comprensión de ideas. La estructura de nodos y estructura de enlaces integran una red de ideas en la base de conocimiento. Es más probable que los estudiantes aprendan más al construir materiales instruccionales que sólo estudiar de ellos.

- $\quad N v u$. Editor de documentos hipertextos (http://www.nvu.com).

- Herramientas para la construcción de páginas Web (http://www. googlepages.com, http://www.netvibes.com/). 
- Bitácoras, Weblogs o Blogs. Herramientas para publicación de contenidos en Internet (http://www.blogger.com, http://wordpress.org/, http://gabbr. com/).

- Audacity. Editor de archivos de sonido (http://audacity.sourceforge.net/).

- VirtualDub. Editor de archivos de video (http://www.virtualdub.org/).

\section{Herramientas de conversación}

Las nuevas teorías de aprendizaje enfatizan tanto la naturaleza social como la constructivista del proceso de aprendizaje. En el mundo real, aprendemos ante una negociación social de significados, no por ser enseñada sino por ser expuesta y discutida. Existen una variedad de ambientes computacionales síncronos y asíncronos que apoyan este proceso de negociación social.

- Comunicaciones en línea (síncrona): Salas de Chats, mensajeros instantáneos (AMSN Aldo messenger http://amsn.sourceforge.net/, Gaim http://gaim. sourceforge.net/, Meebo http://www.meebo.com/).

- Mensajeros instantáneos basados en el protocolo Jabber (http://www.jabber. org/): Exodus (http://exodus.jabberstudio.org/), Google Talk (http://www. google.com/talk/).

- Comunicación asíncrona: Programas cliente para correo electrónico vía web Squirrelmail (http://www.squirrelmail.org/), foros de discusión, listas de correo, mensajes privados, tableros electrónicos y otros.

\section{Herramientas para trabajo colaborativo}

Existen herramientas en Internet que permiten la construcción social del conocimiento, donde existe colaboración comunitaria (Jonassen, 1996).

- Sistemas Wiki (Wikimedia, http://www.wikipedia.org).

- Sistemas de Portales o comunidades virtuales (PostNuke http://noc. postnuke.com/, Joomla! http://www.joomla.org/).

- Sistemas de Administración de Aprendizaje (Moodle http://www.moodle. org, ATutor http://www.atutor.ca/).

- Sistemasdeadministración decontenidosCMS(http://www.opensourcecms. $\underline{\mathrm{com} /) \text {. }}$

- Trabajo colaborativo de proyectos (http://basecamphq.com/, http:// writeboard.com/). 
- Administradores de marcadores sociales (http://del.icio.us/, http://www. furl.net/, http://www.blinklist.com/, http://shadows.com/)

- Sistemas administradores para compartir fotos en línea (http://www.flickr. com/, http://www.bubbleshare.com/).

- Agendas y calendarios (http://www.kiko.com/, http://calendar.google. com/, http://calendarhub.com/).

\section{CONCLUSIÓN}

Se ha realizado una exposición sobre el uso de la computadora como herramienta para extender las capacidades de la mente y fomentar el pensamiento crítico, creativo y complejo y así lograr un aprendizaje efectivo en el estudiante, para lo cual se presentan las ventajas de utilizar software libre, enlistándose ejemplos de herramientas disponibles para ser descargadas de Internet y complementándose con herramientas gratuitas disponibles en Internet para apoyar cierto tipo de actividades de carácter social de apoyo al proceso de aprendizaje. Lo anterior se realiza con la intencionalidad de brindarle a los profesores información puntual y pertinente al respecto de los tipos de aprendizaje que se pueden desarrollar conociendo un abanico de aplicaciones que pueden ser incorporadas bajo un diseño instruccional pertinente. Es importante entonces mencionar que esto tiene implicaciones directas en el concebir a los aprendices como sujetos capaces de adquirir conocimiento y desarrollar aprendizaje de diferentes formas y no sólo a través de la forma expositora del profesor, por lo que entonces hay un cambio significativo en el entorno educativo respecto a la forma de participación de los agentes (profesor y alumnos) que intervienen de forma directa en el proceso enseñanza-aprendizaje ya que el estudiante se vuelve un sujeto activo y dinámico en la generación de su aprendizaje. Por lo mismo, los métodos para verificar que el aprendizaje se ha dado podrían estar más orientados a determinar niveles de comprensión, así como las formas de evaluación deberían incluir entre otros aspectos, carpetas de trabajo y seguimiento del desempeño del alumno.

\section{REFERENCIAS BIBLIOGRÁFICAS}

Díaz Martínez, S. L. (2004). Propuesta de innovación en formación docente para uso de nuevas tecnologías de la información y la comunicación como apoyo a cursos presenciales.
Tesis para obtener grado de Maestría en Innovación Educativa. México: Universidad de Sonora.

Fundación GNU (1992). Definición de Software Libre. [en línea]. Disponible 
en: http://www.gnu.org/philosophy/ free-sw.es.html [consulta 2006, 7 de mayo]

Harris, R. (1998). Introduction to creative thinking. Virtual Salt. [en línea]. Disponible en: (http://www.virtualsalt. com/crebook1.htm [consulta 2006, 7 de mayo]

Jonassen, D. (1996). Computers in the classroom: mindtools for critical thinking. Columbus, OH: Merrill / Prentice Hall. USA.

Jonassen, D.; Chad, C.; Hsiu-Ping, Y. (1998). Computers as Mindtools, for Engaging Learners in Critical Thinking, TechTrends, v43 n2 p24-32. USA. [en línea]. Disponible en: http://www.coe. missouri.edu/ jonassen/Mindtools. pdf [consulta 2006, 7 de mayo]

Jonassen, D. (2002). Computadores como herramientas de la mente. Eduteka. [en línea]. Disponible en: http://www.eduteka.org/tema mes. php3?TemaID =0012 [consulta 2006, 7 de mayo]

Jonassen, D. (2000). Computers as Mindtools for Schools, Engaging Critical Thinking, 2nd Edition. USA: Merril/Prentice Hall.

Lizárraga, C. (2005). Colección de recursos $y$ herramientas de Software Libre y Código de Fuente Abierta. [en línea]. Disponible en: http://software.fisica. uson.mx [consulta 2005, 7 de abril]

O'Reilly, T. (2005). What is Web 2.o. Design Patterns and Business Models for the Next Generation of Software. [en línea]. Disponible en: http://www. oreillynet.com/pub/a/oreilly/tim/ news/2005/09/30/what-is-web-20. html [consulta 2006, 7 de mayo]

Pennock, N. (2001). El uso de las computadoras en la Universidad del Noroeste. Tesis de Maestría. México: Universidad Virtual del Instituto Tecnológico y de Estudios Superiores de Monterrey.

Polo, J. D. (2005). WWWhat's new?: 100 aplicaciones gratuitas Web 2.o. [en línea]. Disponible en: http://wwwhatsnew.blogspot. com/2005/12/100-aplicacionesgratuitas-web-20.html) [consulta 2006, 7 de mayo]

Scriven, M.; Paul, R. (2004). Defining critical thinking: the critical thinking community. [en línea]. Disponible en: (http://www.criticalthinking.org/ aboutCT/definingCT.shtml [consulta 2006, 7 de mayo]

Stallman, R. (1994). Por qué el software no debe tener propietarios. [en línea]. Disponible en: (http://www.gnu.org/ philosophy/why-free.es.html)[consulta 2006, 7 de mayo]

\section{PERFIL ACADÉMICO Y PROFESIONAL DE LOS AUTORES}

Carlos Lizárraga Celaya, Maestro en Ciencias en Física y Matemáticas, y profesor del programa de Ciencias de la Computación en la Universidad de Sonora. Es responsable institucional de la implementación del proyecto Nuevas Tecnologías de la Información y la Comunicación. Sus principales líneas de trabajo e investigación se enfocan en el desarrollo de aplicaciones sobre Internet y la aplicación de la tecnología de la información y comunicación en educación.

E-mail: carlos@fisica.uson.mx 
Sara Lorelí Díaz Martínez, Maestra en Innovación Educativa y profesora del programa de Ingeniería en Tecnología Electrónica así como del curso Introducción a las Nuevas Tecnologías de la Información y la Comunicación del eje de formación común en la Universidad de Sonora. Coordinadora institucional del espacio educativo nuevas tecnologías de la información y la comunicación. Sus principales líneas de trabajo e investigación son la formación docente para la incorporación de las tic's en el proceso enseñanza-aprendizaje.

E-mail: saraloreli@fisica.uson.mx

DIRECCIÓN DE LOS AUTORES

Proyecto Nuevas Tecnologías de la Información y la Comunicación

Edificio 9Q3 planta alta, ala norte

Interior Campus Universitario

Boulevard Encinas y Rosales sin número

Colonia Centro

Código Postal 83000

Hermosillo, Sonora, México.

Fecha de recepción del artículo: 06/10/06

Fecha de aceptación del artículo: 08/02/07 